\title{
Avaliação da qualidade oleoquímica das sementes de gergelim (Sesamum indicum) e girassol (Helianthus annus)
}

Evaluation of the oleochemical quality of sesame seeds (sesamum indicum) and sunflower (helianthus annus)

Evaluación de la calidad oleoquímica de sésamo (sesamum indicum) y girasol (helianthus annus)

Recebido: 18/05/2021 | Revisado: 27/05/2021 | Aceito: 30/05/2021 | Publicado: 12/06/2021

Valentina Ribeiro Castro

ORCID: https://orcid.org/0000-0002-2012-9464 Instituto Federal de Educação, Ciência e Tecnologia do Piaú, Brasil E-mail: valentinarcastro@hotmail.com

Maria Clara dos Santos Furtado ORCID: https://orcid.org/0000-0002-7357-0894 Instituto Federal de Educação, Ciência e Tecnologia do Piauí, Brasil E-mail: clarafurtado@outlook.com

Victória Maura Silva Bermúdez ORCID: https://orcid.org/0000-0002-8105-8735 Universidade Federal do Ceará, Brasil E-mail: vmsbermudez@gmail.com

Edilene Ferreira da Silva

ORCID: https://orcid.org/0000-0001-7073-7962 Universidade Federal de Santa Catarina, Brasil E-mail: silvaedilene16@hotmail.com

Vera Lúcia Viana do Nascimento

ORCID: https://orcid.org/0000-0003-0071-3863 Instituto Federal de Educação, Ciência e Tecnologia do Piauí, Brasil E-mail: veravnascimento@gmail.com

\begin{abstract}
Resumo
Os óleos vegetais são de extrema importância para o organismo humano, sendo considerados fonte de energia e de vitaminas lipossolúveis (A, D, E e K), e fundamentais na promoção de sabor dos alimentos. O óleo de girassol é obtido da semente da planta Helianthus annuиs L., da família Compositae, destacando-se por ser a quarta oleaginosa em produção de grãos e a quinta em área cultivada no mundo. Outra valiosa semente utilizada para extração de óleo é o gergelim (Sesamum indicum, L.), que pertence à família Pedaliaceae. Devido à qualidade e características de alguns óleos vegetais, que podem apresentar alterações após longos períodos de armazenamento, dando-se assim a relevância de estudos à cerca da determinação dessas modificações. A partir disso, este trabalho teve o objetivo de avaliar a qualidade do óleo de gergelim e girassol, comercial e extraído à frio, para obtenção de mais informações sobre as alterações ocorridas durante o período de armazenamento e seu potencial oxidativo. As análises oleoquímicas foram realizadas utilizando-se as variáveis referentes aos índices de iodo, de peróxido, de acidez e \% de ácidos graxos livres (AGL), onde as análises foram realizadas em triplicatas. Como resultado, os óleos de gergelim e girassol comercializados apresentaram valores acima dos limites preconizados pela legislação $\mathrm{RDC} \mathrm{n}^{\circ} 270$, de 22 de setembro de 2005, em relação aos índices oleoquímicos de gergelim e girassol de extração fria apresentaram valores adequados. A contribuição do trabalho desenvolvido evidencia que os óleos após extração, processamento industrial e dependendo de condições do armazenamento podem apresentar a vulnerabilidade e comprometer sua qualidade.
\end{abstract}

Palavras-chave: Ácidos graxos; Alterações lipídicas; Oleaginosas; Óleos vegetais.

\begin{abstract}
Vegetable oils are extremely important for the human organism, being considered a source of energy and of fatsoluble vitamins (A, D, E and K), and fundamental in promoting the taste of food. Sunflower oil is obtained from the seed of the plant Helianthus annuus L., of the Compositae family, standing out for being the fourth oilseed in grain production and the fifth in cultivated area in the world. Another valuable seed used for oil extraction is sesame (Sesamum indicum, L.), which belongs to the Pedaliaceae family. Due to the quality and characteristics of some vegetable oils, which may change after long periods of storage, thus giving relevance to studies on the determination of these changes. From this, this work had the objective of evaluating the quality of the sesame and sunflower oil, commercial and cold extracted, to obtain more information about the alterations occurred during the storage period and its oxidative potential. The oleochemical analyzes were performed using the variables related to the iodine, peroxide, acidity and $\%$ free fatty acids (FFA) indices, where the analyzes were performed in triplicates. As a result,
\end{abstract}


the commercialized sesame and sunflower oils showed values above the limits recommended by the RDC No. 270, of September 22, 2005, in relation to the cold extracting sesame and sunflower oleochemical indexes presented adequate values. The contribution of the work developed shows that oils after extraction, industrial processing and depending on storage conditions can present vulnerability and compromise their quality.

Keywords: Fatty acids; Oilseeds; Lipid changes; Vegetable oils.

\section{Resumen}

Los aceites vegetales son de suma importancia para el organismo humano, siendo considerados una fuente de energía y de vitaminas liposolubles (A, D, E y K), y fundamentales para promover el sabor de los alimentos. El aceite de girasol se obtiene de la semilla de la planta Helianthus annuus L., de la familia Compositae, destacándose por ser la cuarta oleaginosa en producción de cereales y la quinta en superficie cultivada en el mundo. Otra semilla valiosa utilizada para la extracción de aceite es el sésamo (Sesamum indicum, L.), que pertenece a la familia Pedaliaceae. Por la calidad y características de algunos aceites vegetales, que pueden cambiar tras largos periodos de almacenamiento, dando así relevancia a los estudios sobre la determinación de estos cambios. A partir de esto, este trabajo tuvo como objetivo evaluar la calidad del aceite de sésamo y girasol, comercial y extraído en frío, para obtener más información sobre las alteraciones ocurridas durante el período de almacenamiento y su potencial oxidativo. Los análisis oleoquímicos se realizaron utilizando las variables relacionadas con los índices de yodo, peróxido, acidez y\% de ácidos grasos libres (AGL), donde los análisis se realizaron por triplicado. Como resultado, los aceites de sésamo y girasol comercializados mostraron valores por encima de los límites recomendados por el RDC No. 270 , del 22 de septiembre de 2005, en relación a los índices oleoquímicos de sésamo y girasol extractores en frío presentaron valores adecuados. El aporte del trabajo desarrollado muestra que los aceites después de la extracción, el procesamiento industrial y dependiendo de las condiciones de almacenamiento pueden presentar vulnerabilidad y comprometer su calidad.

Palabras clave: Aceites vegetales; Ácidos grasos; Lípidos; Semillas oleaginosas.

\section{Introdução}

Uma crescente preocupação com a saúde, com a qualidade de vida por parte dos consumidores e com a melhoria financeira dos brasileiros tem levado o Brasil a aproveitar sua própria matéria-prima para elaborar produtos inovadores, e minimizar as importações de óleos vegetais utilizados em produtos alimentícios.

De acordo com a Resolução No 49, de 22 de dezembro de 2006, os óleos vegetais são definidos como produtos constituídos principalmente de glicerídeos e ácidos graxos, obtidos unicamente de matéria-prima vegetal refinado, mediante ao emprego adequado de processos tecnológicos avançados. O processamento também pequenas quantidades de outros lipídios, tais como fosfolipídeos, constituintes insaponificáveis e ácidos graxos livres, naturalmente presentes no óleo vegetal. Estas impurezas são eliminadas pelo tratamento do refino e da clarificação do óleo após a extração, uma vez que seu consumo doméstico atende a um controle de qualidade.

Os óleos vegetais são de extrema importância para o organismo humano, e são considerados fonte de energia, veículos de vitaminas lipossolúveis (A, D, E, e K), e fundamentais na promoção de sabor aos alimentos, quando consumidos adequadamente. Apresentam-se na forma líquida à temperatura ambiente de $25^{\circ} \mathrm{C} \pm 2$ e podem ser extraídos de oleaginosas através dos diversos métodos e de diferentes matérias-primas, sendo as mais comuns o óleo de soja, milho, canola, girassol e gergelim (Mandarino et al., 2005).

Atualmente o gergelim é a oleaginosa mais plantada no mundo, tendo alto valor nutritivo, e constitui uma opção como alternativa de renda, fonte de proteína para o consumo humano e enriquecimento de outros produtos (Queiroga et al.,2010). É cultivado em 71 países, especialmente na Ásia e África. A produção mundial está estimada em 3,16 milhões de toneladas (Sluszz \& Machado, 2006). Os maiores produtores mundiais de girassol atualmente são a Rússia, Argentina, Europa Ocidental e Oriental, China e Estados Unidos (CONAB, 2017). Por isso essa cultivar é de fundamental importância econômica.

O óleo de girassol, obtido da semente da planta Helianthus annuus L., da família Compositae, destaca-se por ser a quarta oleaginosa em produção de grãos e a quinta em área cultivada no mundo. O óleo de girassol é interessante pelo seu conteúdo em ácido linoléico. É leve no sabor e aparência e fornece mais vitamina E do que qualquer outro óleo vegetal. É uma 
combinação de gorduras monoinsaturadas e poli-insaturadas com baixos níveis de gordura saturadas, importantes para boa nutrição humana.

O consumo do óleo tem sido associado com os efeitos positivos sobre a saúde humana, devido à ácidos graxos específicos, que possuem os benefícios cardiovasculares, com reconhecidas funções de hidratação e reestruturação, sendo utilizado no tratamento do colesterol e da aterosclerose (Madhavi et al., 2010). Diante desta contextualização, a qualidade e características de alguns óleos podem apresentar alterações, após longos períodos de armazenamento, demonstrando que esta pesquisa é relevante acerca da ocorrência das alterações químicas, que podem contribuir para manter os óleos com características químicas e nutricionais próprias para consumo.

Este trabalho teve o objetivo de avaliar as características óleoquímicas do óleo de gergelim e de girassol extraídos comercializados em rede de supermercados, para que sejam obtidas as informações sobre alterações ocorridas durante os processos e no período de armazenamento.

\section{Metodologia}

A pesquisa foi realizada de forma experimental quantitativa com a utilização de técnicas matemáticas (Pereira et al., 2018). As amostras aleatórias de óleo de gergelim branco (Sesamum indicum) foram adquiridas do comércio foram adquiridas de um pequeno produtor na cidade de Batalha-Piauí, localizada à latitude $04^{\circ} 01^{\prime} 30^{\prime \prime}$ sul e à longitude $42^{\circ} 04^{\prime} 30^{\prime \prime}$ oeste, com altitude de 150 metros (Figura 1). O óleo de gergelim industrializado foi produzido na cidade de São Paulo - SP, e acondicionado em embalagem plástica transparente de $100 \mathrm{ml}$, com prazo de validade até fevereiro de 2021. Já a amostra de óleo de girassol (Helianthus annus) industrializado foi produzido na cidade de Itumbiara - GO, e acondicionado em embalagem plástica transparente de $900 \mathrm{ml}$, dentro do prazo de validade. Ambos foram acondicionados em local arejado e seguro e transportado de forma adequada para o laboratório de Tecnologia de Produtos de Origem Vegetal do Instituto Federal do Piauí, Campus Teresina Central, para realização da extração e analises. As sementes de girassol (Helianthus annus) foram adquiridas no mercado central da cidade de Teresina-Piauí, única capital da Região Nordeste que não se localiza no litoral, distando $343 \mathrm{~km}$ do Oceano Atlântico. Todas as amostras foram acondicionadas em ambiente arejado e seguro para realização da extração dos óleos e análises. Através destas o óleo foi obtido por extração fria, pelo método de Bligh e Dyer (1959).

\section{Preparação das Sementes de Girassol (Helianthus annus) e de Gergelim (Sesamum indicum)}

Para realizar a extração do óleo foi necessário a retirada da casca que envolve as sementes e com utilização de ferramentas de madeiras, espátulas, e tabuas de plástico. As cascas foram quebradas e a semente foi retirada com o uso da espátula. As Figuras 1, 2, 3 e 4 apresentam as etapas de preparação das sementes antes de iniciar o processo de extração do óleo. 
Figura 1 - Sementes de Gergelim (Sesamum indicum).

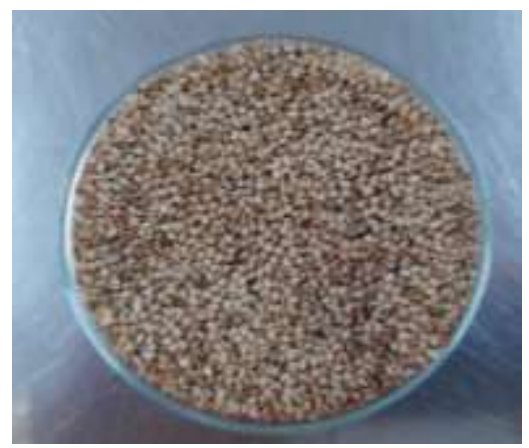

Fonte: Autores (2021)

Figura 3 - Sementes de girassol após secagem.

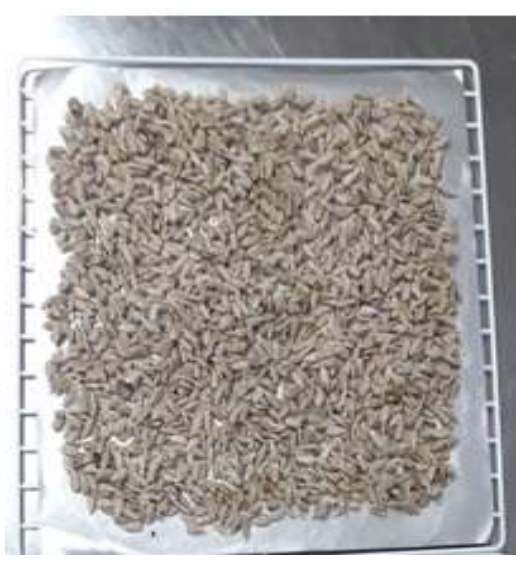

Fonte: Autores (2021)
Figura 2 - Sementes de Girassol (Helianthus annuиs).

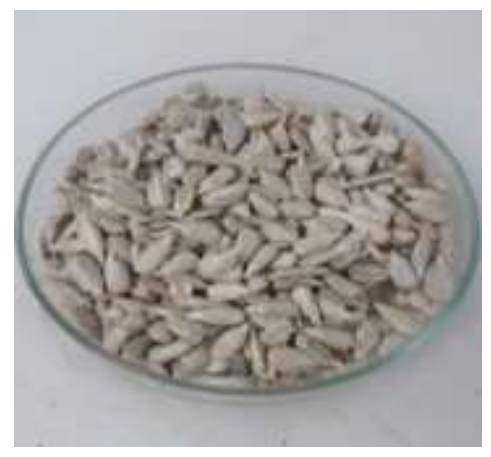

Fonte: Autores (2021)

Figura 4 - Sementes de gergelim após secagem.

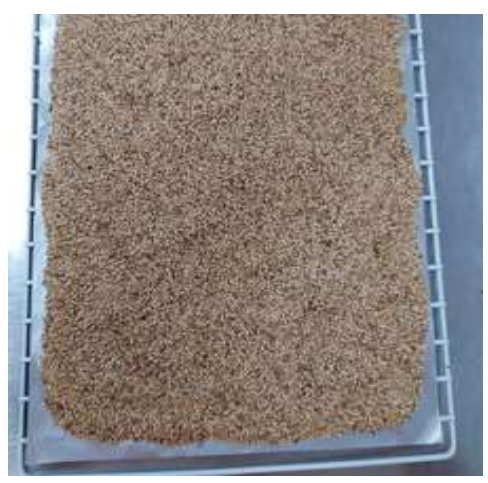

Fonte: Autores (2021)

Na Figura 1 é possível observar as sementes de gergelim in natura e na Figura 2 as sementes de girassol, ambas ainda com casca. Já na figura 3 as sementes já secas de girassol e na Figura 4 as sementes de gergelim já prontas para iniciar a extração do óleo das sementes.

\section{Delineamento experimental}

O planejamento experimental foi do tipo casualizado, onde as amostras de óleos vegetais foram analisadas em triplicatas. O estudo envolveu quatro tratamentos, para os dois óleos, sendo 2 amostras comerciais e 2 amostras naturais, totalizando 04 tratamentos, realizou-se 12 análises em 44 amostras. Cada tratamento foi realizado com três repetições.

Nas amostras analisadas foram verificados os parâmetros oleoquímicos, conforme o Official methods and recommended practices of the American Oil Chemists' Society (AOCS, 2004), que incluíram os seguintes índices: Índices de Acidez (IA), Índice de Ácidos Graxos Livres (\% AGL), Índice de Peróxido (IP). Índice de Iodo (II), conforme a Tabela 1. 
Tabela 1 - Parâmetros oleoquímicos analisados em óleos vegetais.

\begin{tabular}{|c|c|c|}
\hline PARÂMETROS & UNIDADE & MÉTODO \\
\hline Acidez & $\mathrm{mg} \mathrm{NaOH} / \mathrm{g}$ & AOCS $c d 1 c-85$ \\
\hline Ácidos Graxos Livres (AGL) & mg do ácido predominante/100 mg & AOCS Ca 5a-406 \\
\hline Ìndice de Peróxido & $\mathrm{mEq} \mathrm{O}_{2} / \mathrm{kg}$ & AOCS Cd 8-53 \\
\hline Índice de iodo & $\mathrm{g} \mathrm{I}_{2} / 100 \mathrm{~g}$ & AOCS $1 b-87$ \\
\hline
\end{tabular}

Fonte: Adaptado de De Oliveira et al. (2021) e Nascimento et al. (2015).

\section{Extração do Óleo das Sementes de Girassol (Helianthus annuus) e Gergelim (Sesamum indicum)}

O processo de extração de óleo foi iniciado com a lavagem das sementes de gergelim (Sesamum indicum) e girassol (Helianthus annus) com água destilada e secagem no desidratador, e aerador de alimentos Pratic Dyer a $60^{\circ} \mathrm{C} \pm 2$ por 2 horas. Em seguida as sementes foram trituradas em um liquidificador industrial Arno inox e pesadas em balança semi-analítica Bel Engineering, sendo 227,44 g de gergelim. Dessa quantidade foi retirada $50 \mathrm{~g}$ de cada semente, e colocada em um béquer de $500 \mathrm{~mL}$. Neste, juntamente com as sementes foram adicionados $200 \mathrm{~mL}$ de metanol, $50 \mathrm{~mL}$ de clorofórmio, e $100 \mathrm{~mL}$ de água destilada, em uma capela.

Todo o conteúdo foi preservado em refrigerador Carel Easy, por $48 \mathrm{~h}$ a temperatura de $10{ }^{\circ} \mathrm{C} \pm 2$ no recipiente de vidro. Esta etapa foi seguida com a filtração do material e com auxílio de um funil colocado no decantador de 2 L; adicionouse mais $30 \mathrm{~mL}$ de clorofórmio e $40 \mathrm{~mL}$ de água destilada, e deixando decantar por $24 \mathrm{~h}$. Após a decantação, foi realizada a separação de fases, ficando somente a parte oleosa de interesse, que foi retirada e colocada no banho maria, a $61{ }^{\circ} \mathrm{C} \pm 2$ por $2 \mathrm{~h}$. Posteriormente, foi adicionada uma quantidade suficiente de sulfato de sódio anidro $\left(\mathrm{Na}_{2} \mathrm{SO}_{4}\right)$, para retirar o excesso da umidade no óleo, e após isso foi colocado em um frasco de vidro $(250 \mathrm{~mL})$ de cor âmbar armazenado sob refrigeração à 10 ${ }^{\circ} \mathrm{C} \pm 2$. Posteriormente este processo do óleo de gergelim foi submetido à rota-evaporação no equipamento Rotaevaporador Fisatom à temperatura de $65{ }^{\circ} \mathrm{C} \pm 2$, por $1 \mathrm{~h}$, para retirada de reagentes que ainda poderiam estar presentes no óleo. A rotaevaporação foi realizada no Laboratório de Química Analítica, Inorgânica e físico-química do Departamento de química do Instituto Federal do Piauí.

A Figura 5 mostra as etapas dos processos físico-químicos dos tratamentos das oleaginosas de girassol e gergelim para produção de óleos, que correspondem as suas extrações. Inicialmente foi realizada a lavagem das sementes com água destilada, e em seguida colocada em placas da secadora a $60^{\circ} \mathrm{C} \pm 2$ por duas horas; as sementes desidratadas foram trituradas em um liquidificador industrial inox até formar uma farinha homogênea de mesma granulação. Foi adicionado $200 \mathrm{~mL}$ de metanol, 50 $\mathrm{mL}$ de clorofórmio, e $100 \mathrm{~mL}$ de água destilada em $50 \mathrm{~g}$ de farinha de cada tipo de semente; em seguida o material foi refrigerado a $10^{\circ} \mathrm{C} \pm 2$ por $48 \mathrm{~h}$; após, será filtrado e decantado. Foi adicionado de $30 \mathrm{ml}$ de Clorofórmio e $40 \mathrm{ml}$ de água destilada; após a separação da fase oleosa, esta foi levada para o banho maria a em um becker de $600 \mathrm{~mL}$ a $61^{\circ} \mathrm{C} \pm 2$ por $2 \mathrm{~h}$. A umidade do óleo foi retirada com sulfato de sódio anidro, colocado em frasco âmbar, e armazenado sob refrigeração; a rotaevaporação das amostras dos dois óleos foi realizada para retirada dos solventes residuais.

Figura 5 - Fluxograma das etapas de extração dos óleos vegetais. 


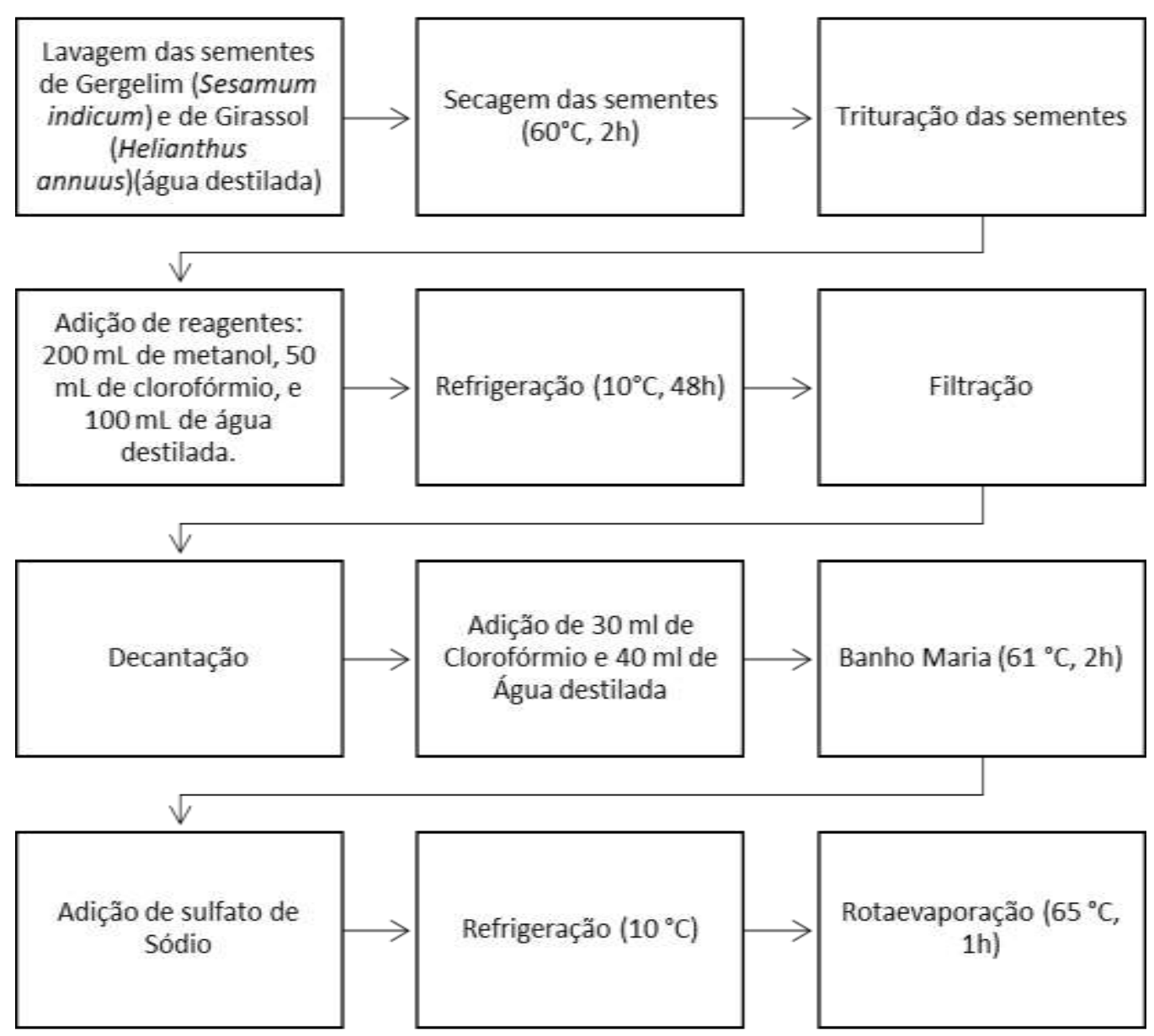

Fonte: Autores (2021)

Após a etapa de rotoevaporação, os óleos já estão disponíveis para realização das análises físico-químico, conforme Figuras 6 e 7.

Figura 6 - Óleo de Girassol (Helianthus annuus)

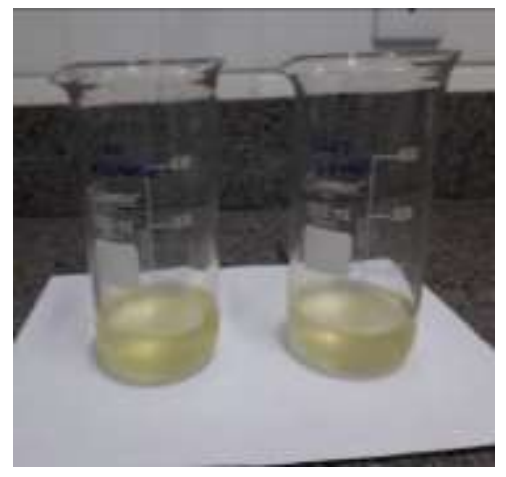

Fonte: Autores (2021)
Figura 7 - Óleo de gergelim (Sesamum indicum)

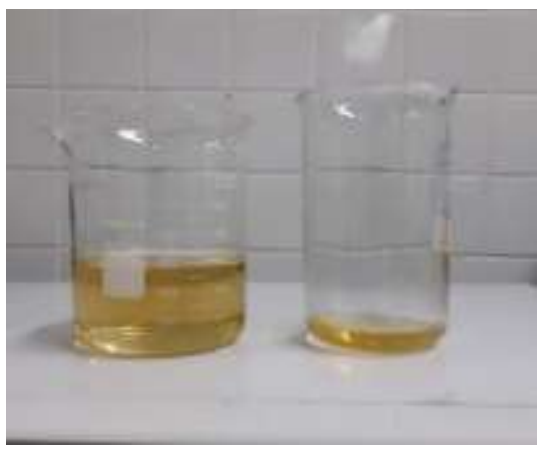

Fonte: Autores (2021) 
Os dados obtidos foram analisados por meio de média \pm SD (desvio padrão). A estatística foi realizada por meio da análise de variância (ANOVA) e comparação pelo Teste de Tukey, e significância estatística estabelecida em 5\%. Os programas utilizados para realizar essa etapa foram Microsoft Excel e Minitab 17 Statistical Software (2016).

\section{Resultados e Discussão}

A Tabela 2 apresenta as análises dos índices de acidez, \% ácidos graxos livres peróxido, e iodo, nas amostras de óleo de gergelim comercializado em supermercados e de extração fria realizada no laboratório de tecnologia de produtos de origem vegetal.

Tabela 2 - Valores médios analisados para os índices de acidez, peróxido, e iodo, nas amostras de óleo de extração fria de gergelim e do óleo comercializado.

\begin{tabular}{lcccc}
\hline \multicolumn{1}{c}{ ÓLEO } & I.A (mg NaOH/g) & $\begin{array}{l}\text { \% AGL }(\mathbf{m g} \\
\text { AG/100mg) }\end{array}$ & I.P (mEq/Kg) & I.I (g I $/ \mathbf{~ 1 0 0 ~ g ) ~}$ \\
\hline $\begin{array}{l}\text { GERGELIM } \\
\text { (Comercial) }\end{array}$ & $5,60^{\mathrm{a}} \pm 0,12$ & $27,99^{\mathrm{a}} \pm 0,60$ & $20,47^{\mathrm{a}} \pm 4,62$ & $201,16^{\mathrm{a}} \pm 4,70$ \\
$\begin{array}{l}\text { GERGELIM } \\
\text { (Extração) }\end{array}$ & $0,88^{\mathrm{b}} \pm 0,15$ & $4,38^{\mathrm{b}} \pm 0,77$ & $5,30^{\mathrm{b}} \pm 1,14$ & $79,64^{\mathrm{b}} \pm 3,80$ \\
\hline
\end{tabular}

Valores médios resultantes das análises de acidez, \% ácidos graxos livres, peroxido e iodo, das amostras de óleo refinado de gergelim comercializado e óleo de extração fria. Médias que não compartilham uma letra na mesma coluna são significativamente diferentes. Fonte: Autores (2021).

Foi verificado que os índices de acidez, \% ácidos graxos livres, de peróxido e de iodo do óleo de gergelim comercial foram significativamente diferentes $(\mathrm{p}<0,05)$ e maiores em relação ao óleo de gergelim de extração fria.

A Tabela 2, mostrou para os óleos de gergelim, que a média dos índices de acidez do óleo comercial $(5,60 \mathrm{NaOH} / \mathrm{g})$ está acima do valor permitido pela legislação, que é de no máximo 0,3 $\mathrm{mgNaOH}$ ( $\mathrm{RDC} \mathrm{n}^{\circ}$ 270, de 22 de setembro de 2005). Em relação ao óleo obtido foi verificado que o valor mais próximo do limite permitido foi de $0,88 \mathrm{mg} \mathrm{NaOH} / \mathrm{g}$. Diversos fatores podem provocar alterações na acidez de um óleo vegetal, a exemplo destes são a umidade do óleo, a exposição à luz e à temperaturas elevadas. Um alto teor de umidade provocaria o aumento da acidez do óleo e a produção da rancidez oxidativa do mesmo, alterando a qualidade do óleo (Almeida et al., 2013). Além disso, o excesso de água aumenta a turbidez do óleo.

Os valores médios de acidez encontrados apresentaram-se semelhantes ao estudo realizado por Antoniassi et al. (2013), que avaliaram sementes de gergelim provenientes de duas cidades diferentes, por meio da extração do óleo com éter de petróleo, sendo possível que fossem encontrados os valores médios de acidez que variaram entre 0,80 e 1,25\%. A quantidade de ácidos graxos livres considerou também um valor maior, indicando, que esses ácidos graxos estavam em maior concentração no referido óleo. De acordo com Mohammed \& Hanza (2008) sobre propriedades de extratos de óleos de Sesamum Indicum L. encontraram valores médios de acidez semelhantes com este estudo, que foram de $0,88 \mathrm{NaOH} / \mathrm{g}$.

Com relação ao óleo de extração fria, a média do índice de peróxido apresentou menor valor médio, ficando dentro da normalidade, ao contrário do gergelim comercial, que está acima do valor exigido pela legislação, que é de no máximo 10 meq/kg para óleos refinados, e de máximo $15 \mathrm{mEq} / \mathrm{kg}$ para óleo de extração a fria. Ao contrário, os altos valores de peróxido $\left(20,47^{\mathrm{a}} \mathrm{mEq} / \mathrm{Kg}\right)$ indicaram que o óleo se encontra em processo oxidativo, ocorrendo a deterioração e alteração de odor e sabor, sendo assim um importante indicador oxidativo utilizado para ser determinado a vida de prateleira de produtos contendo

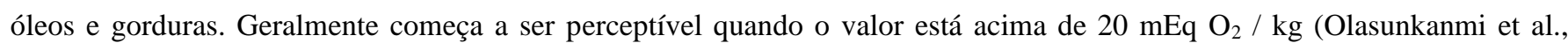


2017). Olasunkami et al., (2017) em seus trabalhos sobre óleo de gergelim relatou um valor de peróxido de $6,0 \mathrm{meq} \mathrm{O}_{2} / \mathrm{kg}$ de óleo. Já Chinweuba e Chendo (2017) encontraram outra variação de valor de peróxido de 4,45 mEq / Kg, sendo este valor inferior ao encontrado nesta pesquisa.

O mesmo estudo mostrou valores médios para o iodo significativamente maiores $(\mathrm{p}<0,05)$ para o óleo comercial de gergelim em relação ao óleo extraído, que se aproximou mais do valor preconizado. A Resolução RDC nº 482, de 23 de setembro de 1999, estabelece valores médios entre 104 - $120 \mathrm{~g} \mathrm{I}_{2} / 100 \mathrm{~g}$, para o óleo de gergelim. De maneira geral, os valores descritos na literatura para os índices de iodo são apresentados como uma faixa de valor ao invés de um número fixo, pois o grau de insaturações pode variar de acordo com aspectos ligados à sazonalidade da oleaginosa, variedade agronômica ou em função do tipo de processamento do óleo (Silva \& Silva, 2016). Valores elevados de iodo tornam o óleo susceptível a degradação térmica e oxidativa, pois quanto maior o grau de insaturação, maior o índice de iodo (De Oliveira et al., 2021). Em estudos realizados por Chinweuba e Chendo (2017), foram encontrados com a análises de iodo, valores semelhantes de 76,56 /100g, para óleo de gergelim. O óleo de gergelim pertence ao grupo de ácido oleico-linoléico (Antoniasse et al, 2013), possui menos que $20 \%$ de ácidos graxos saturados, constituindo-se, principalmente, de ácido palmítico e ácidos esteáricos (Gharby et al., 2017). Olasunkanmi, Omolayo e Olusegun (2017) estudaram perfil de ácidos graxos, propriedades físico-químicas e funcionais de óleo extraídos do gergelim, e foi encontrado valor médio para peróxido de $6,0 \mathrm{mEq} \mathrm{O} / \mathrm{kg}$, considerado inferior aos obtidos nesta pesquisa.

A Tabela 3 apresenta os valores médios resultantes das análises dos índices de acidez, de peróxido e de iodo, nas amostras de óleo de girassol comercializado e de extração fria.

Tabela 3 - Análises dos índices de acidez, de peroxido e de iodo nas amostras de óleo de girassol extraído e do óleo comercializado.

\begin{tabular}{ccccc}
\hline ÓLEO & I.A (mg NaOH/g) & $\begin{array}{c}\text { \% AGL }(\mathbf{m g} \\
\mathbf{A G} / \mathbf{1 0 0} \mathbf{m g})\end{array}$ & I.P $(\mathbf{m E q} / \mathbf{K g})$ & I.I $(\mathbf{g ~ I} \mathbf{2} / \mathbf{~ 1 0 0} \mathbf{g})$ \\
\hline $\begin{array}{c}\text { GIRASSOL } \\
\text { (Comercial) }\end{array}$ & $0,79^{\mathrm{a}} \pm 0,003$ & $3,94^{\mathrm{a}} \pm 0,01$ & $15,89^{\mathrm{a}} \pm 1,98$ & $: 46,30^{\mathrm{a}} \pm 43,82$ \\
$\begin{array}{c}\text { GIRASSOL } \\
\text { (Extração) }\end{array}$ & $1,58^{\mathrm{b}} \pm 0,262$ & $7,89^{\mathrm{b}} \pm 1,31$ & $4,64^{\mathrm{b}} \pm 2,29$ & $79,51^{\mathrm{b}} \pm 16,65$ \\
\hline
\end{tabular}

Valores médios resultantes das análises de acidez, \% ácidos graxos livres, peróxido e iodo, das amostras de óleo de girassol refinado comercializado e de extração fria. Médias que não compartilham uma letra na mesma coluna são significativamente diferentes. Fonte: Autores (2021).

Foi observado que as médias de todos os índices para o óleo de girassol comercial encontravam-se diferenças significativas $(\mathrm{p}<0,05)$ e maiores, quando comparados aos índices do óleo de girassol obtido por extração fria. O índice de acidez do óleo comercializado $(0,79 \mathrm{mg} \mathrm{NaOH} / \mathrm{g})$ está acima dos valores estabelecidos pela legislação de óleos vegetais (ANVISA) e Codex Alimentarius (2019). Enquanto que o óleo extraído de girassol (1,58) encontrou-se dentro do limite estabelecido para acidez, e pode estar relacionada com as diferentes espécies de cultivar, composição do óleo, com o processamento e, principalmente, com as condições de conservação do óleo (Mendonça et al., 2008).

O valor médio do índice de peróxido para o óleo de girassol da extração fria foi de 15,89, ficou dentro do limite permitido pela legislação. No entanto, o óleo de girassol comercial apresentou significativamente $(p<0,05)$ dentro do limite aceitável. Estudos de Tasan e Demirci (2011) realizados sobre extração óleo de sementes oleaginosas de girassol, qualidade e características de estabilidade do óleo encontraram valores médios que variaram entre 8,6 e 9,2 $\mathrm{mEq} \mathrm{O}_{2} / \mathrm{Kg}$, considerados valores maiores que os encontrados nesta pesquisa e pode ter indicado mais estabilidade nos processos. Segundo Furtado et al. 
(2020), o uso de aditivo acidulante, como ácido cítrico. pode contribuir para estabilizar o óleo vegetal. Estudos semelhantes de Masuchi et al. (2008) avaliaram também a estabilidade oxidativa do óleo de girassol comercial, onde foram verificadas as variações de peróxidos entre 2,1 mEq/Kg e 13,3 mEq/Kg.

Em ambos os tratamentos realizados para o óleo de girassol, foram encontrados valores médios de iodo acima do exigido pela legislação para o óleo comercial. A Resolução RDC n 482, de 23 de setembro de 1999 preconiza um índice de iodo (Wijs) de 110 - 143 (g I/2/ $100 \mathrm{~g}$ ) para óleo de girassol. Anna et al. (2009) relatou valores de 148 (g I/2/ 100 g) para óleo de girassol bruto entre 125 - $136\left(\mathrm{~g} \mathrm{I}_{2} / 100 \mathrm{~g}\right)$, para óleo de girassol comercial. Enquanto que pesquisas realizadas por Mohammed e Hamza obtiveram valores maiores (103 e $116 \mathrm{~g} \mathrm{I}_{2} / 100 \mathrm{~g}$ ) que os dados deste estudo.

\section{Conclusão}

Podemos concluir que esta pesquisa mostrou que os óleos de gergelim e girassol comercializados apresentaram valores acima dos limites preconizados pela legislação RDC n ${ }^{\circ} 270$, de 22 de setembro de 2005, em relação aos índices analisados dos óleos de gergelim e girassol de extração fria, podendo esses eventos estarem relacionados ao armazenamento e conservação inadequados ou uso de acidulantes adicionados aos óleos vegetais, uma vez que mostraram características de qualidade preservadas do mesmo pela extração fria do óleo e com poucos danos observados.

É do interesse da produção industrial nacional e internacional de óleos de oleaginosas de girassol e gergelim destinada ao mercado consumidor manter as características físico-químicas dos óleos vegetais, para reduzir a peroxidação, baixar a acidez e ainda mostrar o grau da riqueza da insaturação dos ácidos graxos, mesmo após os processos químicos e físicos. Deve ser contínua a contribuição dos tratamentos metodológicos adequados para as matérias-primas destinadas à elaboração de diversos produtos e àqueles inovados a partir da extração. Os óleos vegetais são ricos em insaturação de ácidos graxos e têm importância nutricional para consumo na alimentação. O consumo do óleo tem sido associado aos efeitos positivos sobre a saúde humana, devido à ácidos graxos essenciais, que possuem os benefícios cardiovasculares, bem como em outras patologias.

Por tanto, mais pesquisas são necessárias para que essas oleaginosas possam ser aproveitadas ao máximo e assim contribuir com o setor agrícola e industrial. Bem como, outros parâmetros podem ser avaliados para melhor avaliação das características

\section{Agradecimentos}

Ao Laboratório de Química Analítica, Inorgânica e físico-química do Departamento de química do Instituto Federal do Piauí (IFPI) localizado no campus Teresina central, Brasil.

\section{Referências}

AOCS - American Oil Chemists' Society. (2009). Official methods and recommended practices of the American Oil Chemists' Society. Champaign, AOCS Anna, L., Pighinelli, K., Park, J., Anna, M. \& Rafael, A. (2009). Otimização da prensagem de grãos de girassol e sua caracterização. Revista Brasileira de Engenharia Agrícola e Ambiental, 13 (1), 63-67.

Almeida, K., Medeiros, E., Gomes, Josivanda, P., Souza, Elisabete, P. \& Santos, J. (2013). Caracterização físico-química de misturas de óleos vegetais para fins alimentares. Revista Verde de Agroecologia e Desenvolvimento Sustentável, 8(1), 218 - 222.

Antoniassi, R., Arriel, N., Gonçalves, E., Freitas, S., Zonolto, D. \& Bizzo, H. (2013). Influência das condições de cultivo na composição da semente e do óleo de gergelim. Revista Ceres, 60 (3), 301-310.

Bligh, E. G. \& Dyer, W. J. (1959). A rapid method of total lipid extraction and purification. Canadian Journal of Biochemistry and Physiology, 37 (8), 911 917.

Brasil. Resolução N ${ }^{\circ}$ 482, de 23 de setembro de 1999. Regulamento Técnico para Fixação de Identidade e Qualidade de Óleos e Gorduras Vegetais. Brasília, DF, DOU no 196-E, 13/10/1999. 
Brasil. Resolução N N9 $^{\text {4 de }} 22$ de dezembro de 2006. Regulamento Técnico de Identidade e Qualidade dos Óleos Vegetais Refinados; a Amostragem; os Procedimentos Complementares; e o Roteiro de Classificação de Óleos Vegetais Refinados, Brasília, DF, D.O.U., 26/12/2006.

Chinweuba, A. \& Chendo, M. (2017). Extraction, Characterisation and Industrial Applications of Sesamum indicum Seed Oil. Modern Chemistry Applications, 5, 216-220.

Codex Alimentarius. Fats, Oils and Related Products. .

CONAB. Companhia Nacional de abastecimento. < https://www.conab.gov.br/>

De Oliveira, A. et al. (2021). Avaliação físico-química do óleo extraído de amendoim (Arachis hypogaea). Research, Society and Development, 10 (1), e49710112011.

FAO - Food and Agriculture Organization of the United Nations (2012) FAOSTAT Production Crops. < http://faostat3.fao.org/home/\#DOWNLOAD>

Furtado, M. et al. (2020) Caracterização físico-química dos óleos do fruto seco de coco (Cocos nucifera L.). Research, Society and Development, 9 (9), e301997211.

Gharby, S. et al. (2017). Chemical characterization and oxidative stability of seeds and oil of sesame grown in Morocco. Journal of the Saudi Society of Agricultural Sciences, 16, 105-111.

Mandarino, J., Roessing, A. \& Benass, V. (2005) Óleos: alimentos funcionais. Embrapa Soja, 91-130.

Madhavi, B. \& Devi, N.K. \& Mrudula, Sai \& Babu, R. (2010). The importance of biodegradable bio-oil-sunflower. International Journal of PharmTech Research. 2. 1913-1915.

Masuchi, M. et al. (2008) Quantificação de tbhq (terc butil hidroquinona) e avaliação da estabilidade oxidativa. Química Nova, 31 (5), $1053-1057$.

Mendonça, M. (2008). Alterações físico-químicas em óleos de soja submetidos ao processo de fritura em unidades de produção de Refeição no Distrito Federal. Revista Comunicação em Ciências da Saúde, 19 (2), 115-122.

Mohammed, M. \& Hamza, Z. (2008). Physicochemical Properties of Oil Extracts from Sesamum Indicum L. Seeds Grown in Jigawa State - Nigeria. J. Appl. Sci. Environ. Manage, 12 (2), $99-101$.

Nascimento, V. et al. (2015). Characterization of a hydrolyzed oil obtained from fish waste for nutraceutical application. Food Science and Technology, 35 (2), 321-325.

Olasunkami, G. et al. (2017). Fatty Acid Profile, Physico-chemical and Funcional Properties of Oil and Protein Isolate Simultaneously Extracted From Sesame (Sesamum indicum) Seed. Food Science and Technology, 18 (1),1-10.

Pereira et al (2018). Methodology of cientific research. UFSM. https://repositorio.ufsm.br/bitstream/handle/1/15824/Lic_Computacao_Metodologia-PesquisaCientifica.pdf?sequence $=1$.

Queiroga, V. et al. (2010). Qualidade fisiológica e composição química das sementes de gergelim com distintas cores. Centro de Ciências Agrárias Universidade Federal de Roraima, Revista Agro Ambiente, 4 (1), 27-33.

Sluszz, T. \& Machado, J. (2006). Características dos potenciais culturas matérias-primas do biodiesel e sua adoção pela agricultura familiar. In Anais: Encontro De Energia No Meio Rural, 6, Campinas.

Silva, C. \& Silva, S. (2016). Avaliação de solventes e condições experimentais para extração do óleo de sementes de nabo forrageiro (Raphanus sativus L.). ENGEVISTA, 18 (2), 348-362.

Tassan, M., Gecgel, U., Demirci, M. (2011). Effects of storage and industrial oilseed extraction methods on the quality and stability characteristics of crude sunflower oil (Helianthus annuus L.). Grasas y aceites, 62 (4), 389-398. 